\title{
COMPACT PERTURBATIONS OF CERTAIN CSL ALGEBRAS
}

\author{
E. G. KATSOULIS AND S. C. POWER \\ (Communicated by Paul S. Muhly)
}

\begin{abstract}
In this note we show that several CSL algebras, including
\end{abstract}

$$
\operatorname{Alg} \mathscr{L}\left(2^{\infty}, \leq, m_{p}\right), \quad 0<p<1,
$$

have compact perturbations that are norm closed.

If $\mathbf{A}$ is an operator algebra acting on a separable Hilbert space $\mathscr{H}$ and $C_{\infty}(\mathscr{H})$ is the ideal of compact operators in $B(\mathscr{H})$, then the algebra

$$
\mathbf{A}+C_{\infty}(\mathscr{H})=\left\{A+K \mid A \in \mathbf{A}, K \in C_{\infty}(\mathscr{H})\right\}
$$

is called the algebra of compact perturbations of $\mathbf{A}$. The study of such algebras has had an important impact both on single operator theory and on the theory of operator algebras. Compact perturbations of nest algebras, the quasitriangular algebras, have been the subject of extensive research and deep results have been obtained by several authors (see $[1,7])$. The fact that these algebras are norm closed was proven by Fall, Arveson, and Muhly [3]. A natural generalization of nest algebras are the CSL algebras; these are reflexive algebras whose invariant subspace lattice consists of mutually commuting projections and is referred to as a CSL. Hopenwasser, Laurie, and Moore [8] extended the result of Fall, Arveson, and Muhly to completely distributive CSL algebras and Gilfeather, Hopenwasser, and Larson [6] to CSL algebras with finite width lattices. On the other hand, Froelich [5] constructed an example of a CSL algebra whose compact perturbations are not norm closed. The purpose of this note is to present new classes of CSL algebras with closed compact perturbations.

We start with the algebras $\operatorname{Alg} \mathscr{L}\left(2^{\infty}, \leq, m_{p}\right), 0<p<1$. These algebras were introduced in [2] and studied in [4, 5, 9]. Several authors have asked if they have closed compact perturbations (see [5, p. 36]). The main reason for such a question is the absence of compact operators in these algebras; all known techniques for showing that certain CSL algebras do have closed compact perturbations depend heavily on the abundance of finite rank operators. In spite of this, in our case one proceeds by estimating the distance of an arbitrary compact operator from $\operatorname{Alg}\left(2^{\infty}, \leq, m_{p}\right), 0<p<1$.

Received by the editors October 26, 1990.

1980 Mathematics Subject Classification (1985 Revision). Primary 47D25; Secondary 47C05.

The first author was supported by the Science and Engineering Research Council. 
Theorem 1. Let $\mathscr{L}$ be a lattice of projections, and let $Q$ be an operator belonging to the weak closure of $\mathscr{L}$, whose spectrum does not contain 0 and 1 . Then for any compact operator $K$ in $B(\mathscr{H})$

$$
\left\|Q^{-1}\right\|^{-1}\left\|(I-Q)^{-1}\right\|^{-1}\|K\| \leq \operatorname{dist}(K, \operatorname{Alg} \mathscr{L}) \leq\|K\| .
$$

Proof. We establish the left inequality since the right one is trivial. First we assume that $K=\sum_{i=1}^{n} e_{i} \otimes f_{i}$ is a finite rank operator. Since $Q$ belongs to the weak closure of $\mathscr{L}$, there exists a sequence $\left\{E_{j}\right\}_{j=1}^{\infty}$ of projections in $\mathscr{L}$ that converges weakly to $Q$. Hence, the sequence $\left\{E_{j}^{\perp}\left(e_{i} \otimes f_{i}\right) E_{j}\right\}_{j=1}^{\infty}$ converges, in the weak operator topology, for all $i$ and thus

$$
\begin{aligned}
\left\|Q^{-1}\right\|^{-1}\left\|(I-Q)^{-1}\right\|^{-1}\|K\| & \leq\|(I-Q) K Q\| \\
& \leq \liminf \left\|E_{j}^{\perp} K E_{j}\right\| \\
& \leq \liminf \left\|E_{j}^{\perp}(A-K) E_{j}\right\| \leq\|A-K\|
\end{aligned}
$$

for any operator $A$ in $\operatorname{Alg} \mathscr{L}$. This proves the result in the case where $K$ is of finite rank. The general case follows from the fact that the distance function is norm continuous.

Corollary 2. Let $\mathscr{L}$ be any noncompact CSL. Then there exists a nonzero projection $P$ in $\mathscr{L}^{\prime \prime}$ such that the algebra $\operatorname{Alg}(P \mathscr{L})+C_{\infty}(P(\mathscr{H}))$ is norm closed.

Proof. Since $\mathscr{L}$ is not compact in the strong operator topology, there exists a positive contraction $Q$ in the weak closure of $\mathscr{L}$ that is not a projection. The Spectral Theorem implies now the existence of a projection $P \in \mathscr{L}^{\prime \prime}$ such that the spectrum of the operator $P Q P$, acting on $P(\mathscr{H})$, does not contain 0 and 1. With this $P$ the result follows; indeed, if $A_{j} \in \operatorname{Alg}(P \mathscr{L}), K_{j} \in C_{\infty}(P(\mathscr{H}))$, and $\left\{A_{j}+K_{j}\right\}_{j=1}^{\infty}$ converges in the norm operator topology then it is a Cauchy sequence. Thus, by Theorem 1 the same true for $\left\{K_{j}\right\}_{j=1}^{\infty}$ and the conclusion follows.

Corollary 3. The algebras

$$
\operatorname{Alg} \mathscr{L}\left(2^{\infty}, \leq, m_{p}\right)+C_{\infty}(\mathscr{H}), \quad 0<p<1
$$

are norm closed.

Proof. Let $\left\{E_{n}\right\}_{n=1}^{\infty}$ be the standard generating family of $\mathscr{L}\left(2^{\infty}, \leq, m_{p}\right)$ (see [2] or [9] for more details). It is easy to check that this sequence converges weakly to the operator $M$, which multiplies every element of $\mathscr{H}$ by the constant function $p$. The result follows now from Theorem 1 and the fact that both $M$ and $I-M$ are invertible.

Remarks. (i) If $\mathscr{L}$ is a (not necessarily commutative) subspace lattice which contains $\mathscr{L}\left(2^{\infty}, \leq, m_{p}\right)$ then $\mathrm{Alg} \mathscr{L}$ has closed compact perturbations. In particular, this applies to a lattice with an Arveson representation $\mathscr{L}(X, \leq, m)$, which arises from an infinite direct product

$$
(X, \leq, m)=\prod_{n=1}^{\infty}\left(X_{n}, \leq_{n}, m_{n}\right)
$$

with $m_{n}$ a nonatomic probability measure (and each $X_{n}$ nontrivial). Indeed using elementary cylinder sets of measure $1 / 2$ leads to a natural identification 
of $\mathscr{L}\left(2^{\infty}, \leq, m_{1 / 2}\right)$ as a sublattice of $\mathscr{L}(X, \leq, m)$. In fact these lattices coincide with infinite tensor products of nonatomic commutative subspace lattices (see [9]).

For another example consider the infinite direct product $(X, \leq, \mu)$ in which each factor is a countable totally ordered set with a faithful measure. Then either $\mathscr{L}(X, \leq, m)$ is purely atomic, or, by the simple grouping argument of $\left[9\right.$, p. 523], there is a sublattice unitarily equivalent to $\mathscr{L}\left(2^{\infty}, \leq, m_{1 / 2}\right)$. It follows that for an arbitrary sequence $\left\{p_{n}\right\}_{n=1}^{\infty}$ in the open unit interval $(0,1)$, the direct product lattice $\mathscr{L}\left(2^{\infty}, \leq, \mu\right)$ for the probability measure $\mu=\mu_{1} \times \mu_{2} \times \cdots$, where $\mu_{k}(\{0\})=p_{k}, \mu_{k}(\{1\})=1-p_{k}$, has a reflexive algebra with closed compact perturbations.

(ii) Theorem 1 suggests the following abstract scheme for constructing noncompact lattices whose algebra has closed compact perturbations. Let $\mathbf{A}$ be any atomless maximal abelian von Neumann algebra and let $A \in \mathbf{A}$ be any positive contraction whose spectrum does not include 0 and 1 . Then there exists a sequence $\left\{E_{j}\right\}_{j=1}^{\infty}$ of projections in A that converges weakly to $A$. If $\mathscr{L}_{A}$ is the lattice generated by $\left\{E_{j}\right\}_{j=1}^{\infty}$, then it is not compact and $\operatorname{Alg} \mathscr{L}_{A}$ contains no nonzero compact operators and has closed compact perturbations.

We now unify the results of Gilfeather, Hopenwasser, and Larson [6] and Hopenwasser, Laurie, and Moore [8]. Except for one modification, our proof follows step by step the proof of Gilfeather, Hopenwasser, and Larson in [6] and we suggest that the reader first reads their proof.

Theorem 4. Let $\mathscr{L}$ be a commutative subspace lattice generated by a completely distributive CSL $\mathscr{L}_{0}$ and finitely many commuting nest $\mathscr{M}_{i}, i=1,2, \ldots, n$. Then the algebra $\operatorname{Alg} \mathscr{L}+C_{\infty}(\mathscr{H})$ is norm closed.

Proof. For convenience we deal only with the case where $\mathscr{L}$ is generated by a completely distributive CSL $\mathscr{L}_{0}$ and a nest $\mathscr{M}$. (In the general case, one argues as in Theorem 5.5 in [6].)

Let $\psi$ be an expectation from $B(\mathscr{H})$ onto $\mathscr{M}^{\prime}$ such that for any operator $X$, the operator $\hat{\psi}(X) \triangleq($ id $-\psi)(X)$ belongs to the ultraweakly closed convex hull of

$\{E X F \mid E, F$ are intervals from $\mathscr{M}, E \ll F$ and $E \perp F\}$.

Let $A_{j} \in \operatorname{Alg} \mathscr{L}, K_{j} \in C_{\infty}(\mathscr{H})$ and assume that $A_{j}+K_{j} \rightarrow T$. We proceed in two steps.

Step 1. $\psi(T)$ belongs to $\mathrm{Alg} \mathscr{L}+C_{\infty}(\mathscr{H})$. Indeed since $\psi$ is norm continuous, $\psi\left(A_{j}\right)+\psi\left(K_{j}\right) \rightarrow \psi(T)$. But $\psi\left(K_{j}\right) \in C_{\infty}(\mathscr{L})$ and $\psi\left(A_{j}\right) \in \mathscr{M}^{\prime} \cap \operatorname{Alg} \mathscr{L}_{0}$ for all $j$ and thus $\psi(T)$ belongs to the norm closure of $\left(\mathscr{M}^{\prime} \cap A \lg \mathscr{L}_{0}\right)+C_{\infty}(\mathscr{H})$. However, Lemma 5.3 in [6] shows that this algebra is closed and so Step 1 is complete.

Step 2. $\hat{\psi}(T)$ belongs to $\operatorname{Alg} \mathscr{L}+C_{\infty}(\mathscr{H})$. In [3] Fall, Arveson, and Muhly define a linear subspace $\mathscr{F}$ to be local if the ultraweak closure of $\mathscr{F} \cap C_{\infty}(\mathscr{H})$ contains $\mathscr{F}$. They then prove that if $\mathscr{F}$ is local and norm closed, then $\mathscr{F}+$ $C_{\infty}(\mathscr{H})$ is norm closed. Hence it is enough to prove that the norm closure of $\hat{\psi}(\operatorname{Alg} \mathscr{L})$ is local. Since $\mathscr{L}_{0}$ is completely distributive, any operator $A$ in $\mathrm{Alg} \mathscr{L}$ is the ultraweak limit of compact operators in $\mathrm{Alg} \mathscr{L}_{0}$ (see [8]) and thus, for every pair $E, F$ of intervals from $\mathscr{M}$ such that $E \ll F$ and $E \perp F$, $E A F$ is the ultraweak limit of compact operators belonging to Alg $\mathscr{L}_{0} \cap A \lg \mathscr{M}=$ 
$\operatorname{Alg} \mathscr{L}$. The properties of $\psi$ guarantee that the same is true for $\hat{\psi}(A)$ and the proof of Step 2 is complete.

The conclusion follows immediately from Steps 1 and 2 .

\section{REFERENCES}

1. N. Andersen, Compact perturbations of reflexive algebras, J. Funct. Anal. 38 (1980), 366400 .

2. W. Arveson, Operator algebras and invariant subspaces, Ann of Math. (2) 100 (1974), 433532.

3. T. Fall, W. Arveson, and P. Muhly, Perturbations of nest algebras, J. Operator Theory, 1 (1979), 137-150.

4. J. Froelich, Compact operators in the algebra of a partially ordered measure space, J. Operator Theory 10 (1983), 353-355.

5. __ Compact operators, invariant subspaces and spectral synthesis, J. Funct. Anal. 81 (1988), 1-37.

6. F. Gilfeather, A. Hopenwasser, and D. R. Larson, Reflexive algebras with finite width lattices: cohomology, tensor products, compact perturbations J. Funct. Anal. 55 (1984), 176-199.

7. D. Herrero, Compact perturbations of nest algebras, index obstructions and a problem of Arveson, J. Funct. Anal. 55 (1984), 78-109.

8. A. Hopenwasser, C. Laurie, and R. Moore, Reflexive algebras with completely distributive subspace lattices, J. Operator Theory 11 (1984), 91-108.

9. B. H. Wagner, Weak limits of projections and compactness of subspace lattices, Trans. Amer. Math. Soc. 304 (1987), 515-535.

Department of Mathematics, LanCASter University, LANCAster LA1 4YL, ENGlaNd 